\title{
O impacto de estratégias de ventilação mecânica que minimizam o atelectrauma em um modelo experimental de lesão pulmonar aguda
}

\author{
The impact of mechanical ventilation strategies that minimize atelectrauma \\ in an experimental model of acute lung injury \\ Mario E. G. Viana1, Guilherme A. Sargentelli2, André L. M. Arruda ${ }^{3}$, \\ Budi Wiryawan ${ }^{4}$, Alexandre T. Rotta ${ }^{5}$
}

\section{Resumo}

Objetivo: Avaliar se estratégias ventilatórias que buscam a estabilização alveolar e a prevenção do atelectrauma estão associadas a desfechos fisiológicos mais favoráveis em um modelo experimental de lesão pulmonar aguda combinada.

Métodos: Trinta e nove coelhos foram instrumentados e ventilados com uma fração inspirada de oxigênio $\left(\mathrm{FiO}_{2}\right)$ de 1,0 . A lesão pulmonar foi induzida pela infusão venosa de lipopolissacarídeo de $E$. coli e por repetidas lavagens traqueais com solução salina. Os animais foram randomizados a receber ventilação mecânica convencional com volume corrente de $10 \mathrm{ml} / \mathrm{kg}$, pressão expiratória final (PEEP) de $4 \mathrm{~cm} \mathrm{H}_{2} \mathrm{O}$; ventilação mecânica convencional com surfactante (Infasurf, $3 \mathrm{mg} / \mathrm{kg}$, ET); ventilação líquida parcial (18 $\mathrm{ml} / \mathrm{kg}$ de perflubron, ET); ou ventilação oscilatória de alta freqüência, com pressão média de via aérea de $14 \mathrm{~cm} \mathrm{H}_{2} \mathrm{O}$ e freqüência de $10 \mathrm{~Hz}$. Animais sadios submetidos a instrumentação e ventilação convencional serviram como controles. Os grupos ventilação mecânica convencional com surfactante, ventilação líquida parcial e controle foram ventilados com parâmetros idênticos ao grupo ventilação mecânica convencional. Os animais foram estudados por 4 horas, durante as quais gasometrias arteriais foram obtidas a cada 30 minutos. Após o sacrifício, os pulmões foram retirados para graduação de lesão através de um escore de dano histológico e dosagem de 4-hidroxi-nonenal, um marcador de peroxidação lipídica.

Resultados: A ventilação mecânica convencional resultou em hipoxemia e lesão pulmonar significativa. Animais tratados com ventilação líquida parcial, ventilação oscilatória de alta freqüência ou ventilação mecânica convencional com surfactante apresentaram oxigenação adequada, mas a ventilação mecânica convencional com surfactante resultou em escores de lesão pulmonar mais elevados e maior dano oxidativo.

Conclusões: Estratégias que minimizam o atelectrauma (ventilação mecânica convencional e ventilação oscilatória de alta freqüência) estão associadas a oxigenação adequada e atenuação da lesão pulmonar. A reposição de surfactante melhora a oxigenação em comparação com a ventilação mecânica convencional, mas resulta em lesão pulmonar aumentada, presumivelmente porque o PEEP inadequadamente baixo foi insuficiente para estabilizar os alvéolos durante a expiração.

J Pediatr (Rio J). 2004;80(3):189-96: Lesão pulmonar aguda, atelectrauma, ventilação mecânica, síndrome do desconforto respiratório agudo, ventilação oscilatória de alta frequeência, ventilação líquida, surfactante.

\begin{abstract}
Objective: To evaluate whether ventilation strategies that target alveolar stabilization and prevention of atelectrauma would be associated with more favorable physiologic outcomes in a combined model of acute lung injury.

Methods: Thirty-nine rabbits were instrumented and ventilated with $\mathrm{FiO}_{2}$ of 1.0. Combined lung injury was induced by an infusion of lipopolysaccharide and tracheal saline lavage. Animals were randomized to receive conventional ventilation with tidal volume of $10 \mathrm{ml} / \mathrm{kg}$, PEEP of $4 \mathrm{~cm} \mathrm{H} \mathrm{H}_{2} \mathrm{O}$; conventional ventilation with surfactant (Infasurf, $3 \mathrm{mg} / \mathrm{kg} \mathrm{IT}$ ); partial liquid ventilation ( $18 \mathrm{ml} / \mathrm{kg}$ of perflubron IT); or high-frequency oscillatory ventilation with mean airway pressure of $14 \mathrm{~cm} \mathrm{H} \mathrm{H}_{2} \mathrm{O}$ and frequency of $4 \mathrm{~Hz}$. Uninjured ventilated animals served as controls. Conventional ventilation with surfactant, partial liquid ventilation and control groups were ventilated with settings identical to the conventional ventilation group. Animals were studied for 4 hours, during which serial blood gas measurements were obtained. After sacrifice, lungs were harvested for injury grading by a microscopic lung injury score and measurement of 4-hydroxy-nonenal, a marker of lipid peroxidation.

Results: Conventional ventilation resulted in hypoxia and greater evidence of lung injury. Animals treated with partial liquid ventilation, high-frequency oscillatory ventilation or conventional ventilation with surfactant had adequate oxygenation, but conventional ventilation with surfactant resulted in higher lung injury scores and increased pulmonary oxidative damage.

Conclusion: Strategies that minimize atelectrauma (partial liquid ventilation and high-frequency oscillatory ventilation) are associated with adequate oxygenation and attenuated lung injury. Surfactant improves oxygenation in comparison to conventional ventilation alone but resulted in increased injury, presumably because the inadequately Iow PEEP was insufficient to stabilize the alveoli during expiration.
\end{abstract}

J Pediatr (Rio J). 2004;80(3):189-96: Acute lung injury, atelectrauma, mechanical ventilation, acute respiratory distress syndrome, highfrequency oscillatory ventilation, liquid ventilation, surfactant.

1. Doutor em Fisiologia Experimental pela Universidade de São Paulo. Diretor Médico, Prontobaby Hospital da Criança, Rio de Janeiro, RJ.

2. Chefe, Unidade de Terapia Intensiva Pediátrica, Prontobaby Hospital da Criança, Rio de Janeiro, RJ.

3. Mestre em Pediatria pela Universidade Federal Fluminense (UFF). Professor de Pediatria, Faculdade de Medicina Souza Marques (FTESM). Médico, UTI Pediátrica, Prontobaby Hospital da Criança, Rio de Janeiro, RJ.

4. Professor assistente de Pediatria, State University of New York at Buffalo, Estados Unidos.

5. Professor assistente de Anestesiologia, University of Texas Medical Branch. Intensivista pediátrico, Driscoll Children's Hospital, Corpus Christi, TX, Estados Unidos. Fontes financiadoras: Núcleo de Pesquisa do Prontobaby Hospital da Criança, Alliance Pharmaceutical Corp., Viasys Healthcare Inc. (SensorMedics).

Artigo submetido em 06.01.04, aceito em 03.03.04. 


\section{Introdução}

Sabe-se que a lesão da unidade alveolocapilar, associada à disfunção microcirculatória, ao acúmulo de fluido alveolar e a anormalidades funcionais do surfactante, faz com que pacientes com lesão pulmonar aguda (LPA) e síndrome do desconforto respiratório agudo (SDRA) apresentem um grave desequilíbrio entre ventilação e perfusão, resultando em hipoxemia bastante significativa ${ }^{1}$. Adicionalmente, o trabalho respiratório exagerado em função do elevado espaço morto e da diminuição da complacência pulmonar leva à falência respiratória, marcada por hipercapnia e acidose respiratória ${ }^{1}$. Como conseqüência, a grande maioria dos pacientes com LPA e SDRA necessita de ventilação mecânica ${ }^{2}$ para estabilizar o quadro respiratório, propiciando, assim, que haja tempo para o tratamento das afecções de base e resolução natural do processo pulmonar. Apesar dos avanços nos cuidados gerais de terapia intensiva, as dramáticas alterações na fisiologia respiratória delineadas acima fazem com que pacientes com LPA e SDRA continuem apresentando mortalidade bastante alta, variando entre 35 e $71 \%{ }^{3-5}$.

Atualmente, a ventilação pulmonar mecânica na LPA e SDRA não pode mais ser encarada como uma mera terapia de suporte, mas sim como uma modalidade terapêutica capaz de influenciar significativamente a evolução da doença pulmonar e o desfecho clínico5,6. Estratégias de ventilação mecânica que empregam volumes correntes elevados, resultando na distensão cíclica exagerada de alvéolos durante a inspiração, estão claramente associadas à progressão da doença pulmonar (volutrauma) em modelos experimentais 7,8 e estudos clínicos ${ }^{5,6}$. Por outro lado, a exata contribuição exercida pelo colapso expiratório cíclico alternado à reabertura inspiratória de unidades alveolares no agravamento da lesão pulmonar (atelectrauma) ${ }^{9}$ é menos óbvia. Apesar da comprovação de que o emprego de estratégias ventilatórias que evitam o atelectrauma - através da aplicação de pressão expiratória final (PEEP) adequada - resulta em atenuação dos marcadores biológicos de lesão pulmonar ${ }^{10,11}$, um recente estudo clínico comparando o uso de PEEP alta e PEEP baixa em pacientes com SDRA foi encerrado por futilidade, devido à ausência de diferenças significativas entre os dois grupos ${ }^{12}$.

Neste trabalho, nosso objetivo foi o de investigar o impacto de estratégias ventilatórias que reduzem ou previnem o atelectrauma, como a ventilação oscilatória de alta freqüência (VOAF), a ventilação líquida parcial com perfluorocarbonos (VLP) e a ventilação mecânica convencional (VMC) associada ao uso do surfactante, em comparação a uma estratégia que sabidamente causa atelectrauma, como a VMC com PEEP inadequadamente baixa ${ }^{11}$, em um modelo combinado de lesão pulmonar aguda. A hipótese testada é a de que estratégias ventilatórias capazes de prevenir o atelectrauma estão associadas a melhor oxigenação, menor evidência de lesão histopatológica e atenuação do dano oxidativo pulmonar.

\section{Materiais e métodos}

Este estudo foi realizado no laboratório experimental da Divisão de Terapia Intensiva Pediátrica, no Centro de Pesquisas Biomédicas da State University of New York at Buffalo, em Buffalo, Nova Iorque, Estados Unidos da América. O protocolo experimental foi aprovado pelo Comitê Institucional de Cuidados e Uso de Animais da própria universidade. Os reagentes químicos utilizados neste estudo foram adquiridos da Sigma Chemical (St. Louis, EUA), exceto quando explicitamente mencionado.

\section{Animais e randomização}

Os animais foram tratados de acordo com as normas publicadas pelo Instituto Nacional de Saúde dos Estados Unidos da América. Trinta e nove coelhos jovens brancos da linhagem Nova Zelândia, com peso corporal entre 1,9 e 2,5 $\mathrm{kg}$, foram utilizados no decorrer deste estudo. Os animais foram randomizados antes da indução anestésica através de um sistema de envelopes idênticos e tinham chances iguais de pertencerem a qualquer um dos cinco grupos experimentais.

\section{Anestesia e sedação}

Os animais foram anestesiados e sedados com uma injeção intramuscular de $25 \mathrm{mg} / \mathrm{kg}$ de cetamina (Fort Dodge Animal Health, Fort Dodge, EUA) e $4 \mathrm{mg} / \mathrm{kg}$ de xilazina (The Butler Company, Columbus, EUA). A pré-oxigenação foi feita durante respiração espontânea de um fluxo de gás contínuo (10 l/min) com $100 \%$ de oxigênio, através de uma máscara facial. Um acesso venoso foi obtido por punção da veia auricular lateral (22 Gauge Jelco, Johnson \& Johnson, Arlington, EUA). O relaxamento muscular foi induzido com a administração intravenosa de $0,2 \mathrm{mg} / \mathrm{kg}$ de pancurônio (Baxter HealthCare Corp., Irvine, EUA) e mantido com doses adicionais de $0,1 \mathrm{mg} / \mathrm{kg}$ a cada hora. A manutenção da anestesia e sedação foi feita pela administração intravenosa contínua de $10 \mathrm{mg} / \mathrm{kg} /$ hora de cetamina e 4 $\mathrm{mg} / \mathrm{kg} /$ hora de xilazina até a conclusão do experimento.

\section{Instrumentação}

Os animais anestesiados foram posicionados em decúbito dorsal, e a região anterior do pescoço foi rapidamente dissecada para a realização de uma traqueostomia. Um tubo traqueal com balonete $(3,0$ a 3,5 mm, Sheridan Catheter, Argyle, EUA) foi introduzido através da incisão traqueal e fixado com fita umbilical. Durante a dissecção, a veia jugular interna e a artéria carótida comum foram isoladas. Um cateter vascular de lúmen único (Intramedic Polyethylene Tubing, Beckton Dickinson, Sparks, EUA) foi introduzido na artéria carótida comum e avançado até a porção intratorácica desse vaso. Um cateter venoso de lúmen duplo (4 French, $8 \mathrm{~cm}$, Cook Incorporated, Bloomington, EUA) foi introduzido até a junção entre o átrio direito e a veia cava superior através da veia jugular interna. Ambos os cateteres foram conectados a um monitor fisiológico (Horizon 2000, Mennen Medical, Clearance, EUA) para a medição contínua de pressão arterial e pressão venosa central. 
A temperatura corporal central foi acompanhada através de um sensor esofágico, e a normotermia foi mantida através do uso de um lençol elétrico aquecido. Fluidos de manutenção foram administrados de maneira contínua, com uma infusão de $4 \mathrm{ml} / \mathrm{kg} /$ hora de solução salina $0,9 \%$ contendo $5 \%$ de dextrose.

Durante o processo de instrumentação, todos os animais foram ventilados com um respirador Servo 300 (Siemens-Elema, Solna, Suécia), com fração inspirada de oxigênio $\left(\mathrm{FiO}_{2}\right)$ de 1,0 , volume corrente de $10 \mathrm{ml} / \mathrm{kg}$, PEEP de $4 \mathrm{cmH}_{2} \mathrm{O}$ e freqüência respiratória de 25 ciclos por minuto. Esses parâmetros foram mantidos por um período de estabilização de 15 minutos até o momento da indução da lesão pulmonar aguda.

\section{Curvas volume-pressão}

Curvas estáticas de volume-pressão do sistema respiratório foram obtidas ao final do período de estabilização e após a indução da lesão pulmonar aguda. A pressão de via aérea foi medida através de um transdutor ( $P 23 \mathrm{XL}$, Spectramed, Ounard, EUA) acoplado lateralmente ao tubo traqueal, com os sinais transmitidos a um gravador fisiológico de múltiplos canais (TA6000, Gould Instruments, Valley View, EUA) ${ }^{11}$.

\section{Indução da lesão pulmonar}

A lesão pulmonar aguda foi induzida pela administração intravenosa de endotoxina ${ }^{13}$ e lavagem pulmonar com solução salina ${ }^{13,14}$. Foram utilizados lipopolissacarídeos de Escherichia coli, reconstituídos em solução salina a uma concentração final de $0,5 \mathrm{mg} / \mathrm{kg}$ e infundidos durante 5 minutos. Esse procedimento foi seguido de lavagem pulmonar por via endotraqueal com três alíquotas de $30 \mathrm{ml} / \mathrm{kg}$ de solução salina aquecida a $38{ }^{\circ} \mathrm{C}^{14}$. Os animais foram estabilizados por 15 minutos, após os quais uma gasometria arterial foi obtida para confirmar o grau de hipoxemia necessário para a continuação do protocolo experimental $\left(\mathrm{PaO}_{2}<100\right.$ torr $)$.

\section{Grupos experimentais}

Os animais do grupo VOAF foram ventilados com o oscilador SensorMedics 3100A (Viasys Healthcare, Yorba Linda, EUA). Todos os demais grupos experimentais foram ventilados com um respirador Servo 300, no modo volume controlado. A $\mathrm{FiO}_{2}$ foi mantida em 1,0 durante a realização da fase de intervenção do experimento, que durou 4 horas. Os animais submetidos a lesão pulmonar foram randomizados a um de quatro grupos experimentais: 1) VMC $(n=7)$ : ventilação com volume corrente de $10 \mathrm{ml} / \mathrm{kg}$, PEEP de $4 \mathrm{~cm} \mathrm{H} \mathrm{H}_{2} \mathrm{O}$, tempo inspiratório de 1 segundo e freqüência respiratória de 25 a 40 ciclos por minuto, ajustada de acordo com a $\mathrm{PaCO}_{2} ; 2$ ) surfactante (VMC-S; $\mathrm{n}=8$ ): ventilação idêntica ao grupo VMC, com a adição de uma dose endotraqueal de $3 \mathrm{mg} / \mathrm{kg}$ de surfactante natural exógeno (Infasurf, ONY, Inc., Amherst, EUA); 3) VLP ( $n=7)$ : ventilação idêntica ao grupo VMC, com a adição de uma dose de $18 \mathrm{ml} / \mathrm{kg}$ de perflubron
(Alliance Pharmaceutical, Corp., San Diego, EUA) por via endotraqueal (a cada hora, estes animais eram desconectados do respirador para observação da presença de um menisco líquido no tubo endotraqueal; na ausência de um menisco líquido, $2 \mathrm{ml} / \mathrm{kg}$ de perflubron eram administrados para reposição de perdas evaporativas); 4) VOAF $(n=8)$ : ventilação com pressão média de via aérea de $14 \mathrm{~cm} \mathrm{H} \mathrm{H}_{2} \mathrm{O}$, tempo inspiratório de $33 \%$, freqüência de 10 $\mathrm{Hz}$ e amplitude necessária para manter a $\mathrm{PaCO}_{2}$ em níveis fisiológicos. Um grupo de animais sadios instrumentados e ventilados de forma idêntica ao grupo VMC foram usados como controles ( $n=9)$.

Gasometrias arteriais foram realizadas no estado basal, após a lesão (nos grupos experimentais) e a cada 30 minutos pelas 4 horas de duração do protocolo de ventilação. Imediatamente antes do término do experimento, os animais receberam uma dose de heparina (100 unidades $/ \mathrm{kg}$, endovenoso) e foram sacrificados com a administração rápida de pentobarbital (100 mg/kg, endovenoso).

\section{Coleta de amostras}

Imediatamente após o sacrifício, o tubo endotraqueal foi ocluído, e o tórax foi cuidadosamente aberto para excluir a presença de um pneumotórax oculto, confirmar a posição ideal de cateteres vasculares e do tubo traqueal e coletar amostras teciduais.

O pulmão direito foi isolado por meio da colocação de uma alça apertada de fita umbilical ao nível do hilo. $O$ átrio esquerdo foi fenestrado, e a artéria pulmonar foi canulada com um cateter para a infusão de $100 \mathrm{ml}$ de solução salina heparinizada. O pulmão esquerdo foi dissecado em cortes axiais que foram rapidamente congelados em nitrogênio líquido e preservados em $-70^{\circ} \mathrm{C}$ para subseqüente análise. O pulmão direito foi dissecado com cortes axiais de $0,5 \mathrm{~cm}$ de espessura, sendo que o corte central (no nível do hilo) foi fixado em formol a $10 \%$ para análise histológica.

\section{Análise do dano oxidativo}

O dano oxidativo pulmonar foi quantificado através dos níveis de 4-hidroxinonenal (4-HNE), um marcador específico de peroxidação lipídica, através do método de Esterbauer \& Cheeseman 15,16 .

\section{Análise histopatológica}

Cortes axiais de pulmão foram corados com hematoxilina e eosina e examinados independentemente por dois investigadores, de forma cega. Em cada lâmina, o espécime era dividido em duas zonas distintas, representando as regiões dependentes (dorsais) e não-dependentes (ventrais) do pulmão. Dez campos microscópicos foram aleatoriamente selecionados para o exame, sendo cinco em cada uma das regiões. Um escore de dano histopatológico pulmonar que analisa sete variáveis com cinco graus individuais de severidade foi utilizado para quantificar objetivamente a magnitude da lesão por meio de microscopia óptica ${ }^{17,18}$. 


\section{Análise estatística}

Os resultados foram expressos como médias e desvio padrão e analisados com o uso do programa SigmaStat 2.03 (SPSS, Chicago, EUA). Variáveis com distribuição normal foram comparadas entre os diversos grupos experimentais através de análise de variância (ANOVA), com subseqüentes comparações múltiplas entre pares através do teste de Student-Newman-Keuls. Variáveis de distribuição não-normal foram comparadas entre os diversos grupos experimentais através de ANOVA de Kruskal-Wallis, com subseqüentes comparações entre pares através do teste de Dunn. A análise do comportamento de uma variável ao longo do tempo foi analisada através de ANOVA para medidas repetidas de Friedman, com subseqüentes comparações múltiplas entre pares através do método de Dunnett. Foi utilizado um nível de significância estatística de 5\% (alfa =0,05).

\section{Resultados}

\section{Alteração de complacência do sistema respiratório}

Todos os animais submetidos a lesão pulmonar por administração de endotoxina intravenosa e lavagem de surfactante apresentaram uma piora significativa da complacência do sistema respiratório, aferida pela medida da relação entre volume e pressão estática no período pósinjúria em comparação com o estado basal (Figura 1). Um ponto de inflexão inferior em aproximadamente 12 a 13 $\mathrm{cmH}_{2} \mathrm{O}$ pode ser visualizado em todos os grupos lesados.

\section{Oxigenação}

A distribuição das medidas de $\mathrm{PaO}_{2}$ para os diferentes grupos experimentais é mostrada na Figura 2. Todos os grupos apresentavam oxigenação normal antes da indução de lesão pulmonar. Imediatamente após a indução de lesão, todos apresentaram uma redução significativa da $\mathrm{PaO}_{2}$ comparativamente ao estado basal. O grupo VMC manteve uma $\mathrm{PaO}_{2}$ significativamente mais baixa que os demais durante todo o experimento. Da mesma forma, o grupo VLP manteve uma $\mathrm{PaO}_{2}$ entre $380 \mathrm{mmHg}$ e 400 $\mathrm{mmHg}$, que, apesar de estatisticamente mais baixa que as dos demais, é compatível com a oxigenação de pulmões normais submetidos a essa modalidade ${ }^{19,20}$. Animais tratados com VMC-S apresentaram melhora gradual de oxigenação no decorrer do experimento, presumivelmente pelo recrutamento alveolar progressivo. $\mathrm{A} \mathrm{PaO}_{2}$ desses animais foi significativamente mais alta do que as dos tratados com VLP aos 210 e 240 minutos de estudo. Animais tratados com VOAF apresentaram os maiores níveis de $\mathrm{PaO}_{2}$ dentre todos os grupos lesados, retornando prontamente a valores compatíveis com o estado prélesão e comparáveis aos encontrados no grupo controle, indicando um recrutamento pulmonar ótimo.

\section{Dano histopatológico}

Os animais do grupo controle apresentaram arquitetura pulmonar normal, em contraste com amostras do grupo VMC, onde tanto a região não-dependente quanto a depen- dente apresentaram denso infiltrado inflamatório, edema proteináceo, hemorragia alveolar e intersticial, e atelectasia (Figura 3). Animais tratados com VOAF, VLP ou VMC-S mantiveram uma arquitetura pulmonar mais preservada comparativamente com os animais tratados com ventilação convencional. Amostras do grupo VOAF apresentaram menos lesão na região não-dependente, com uma tendência a um maior grau de lesão na região dependente. Em contraste, animais tratados com VLP apresentaram maior evidência de lesão na região não-dependente em comparação com a região dependente. Animais tratados com VMC-S tiveram
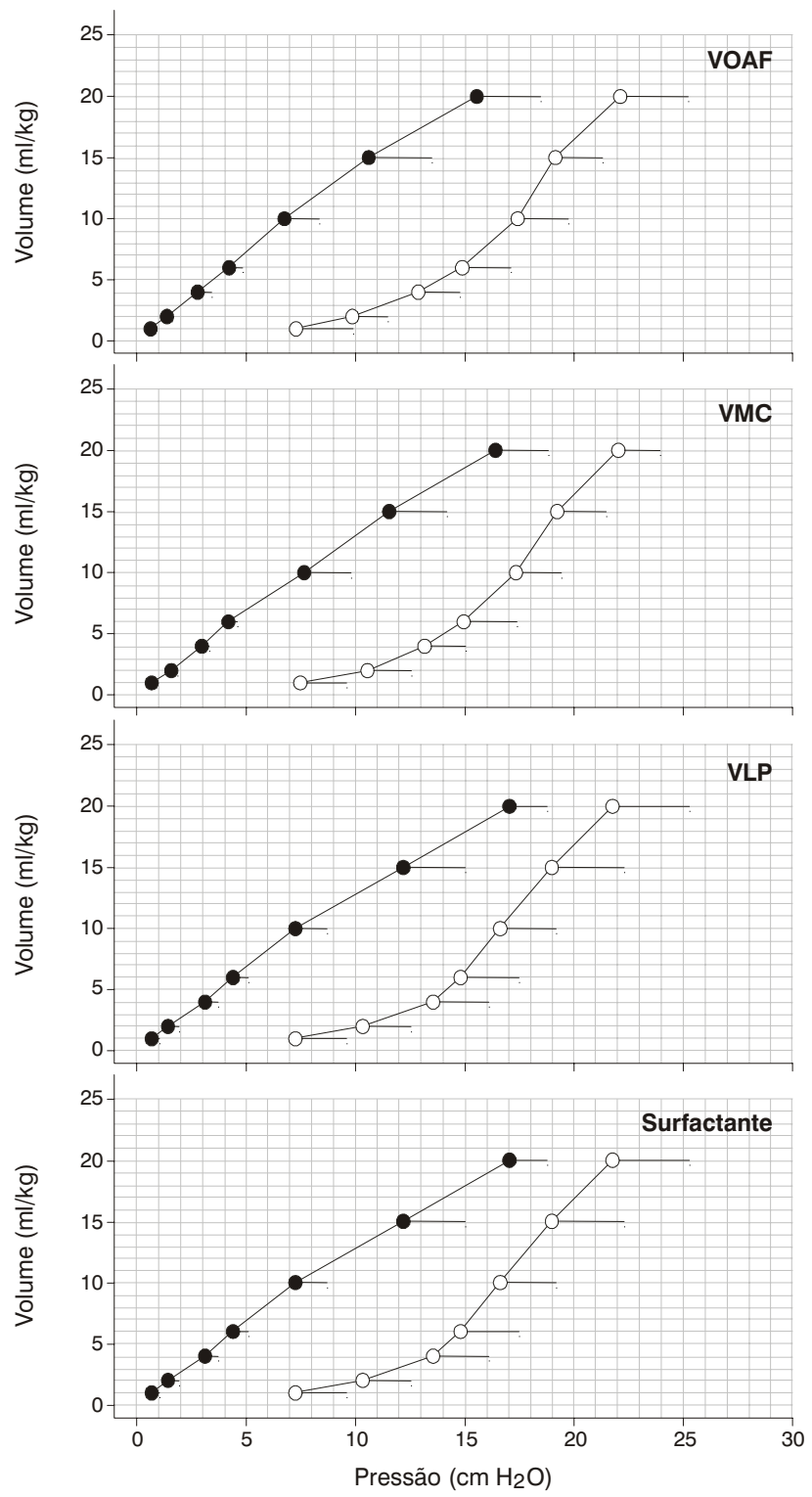

VOAF: ventilação oscilatória de alta freqüência; VMC: ventilação mecânica convencional; VLP: ventilação líquida parcial.

Figura 1 - Relação entre volume e pressão estática de via aérea para animais no estado basal (círculos pretos) e após indução da lesão pulmonar (círculos brancos) 
maior evidência de lesão do que os tratados com VOAF e VLP, apesar de apresentarem menos dano que os animais tratados com VMC. A lesão histológica nos animais tratados com surfactante foi mais pronunciada na amostra da região dependente do pulmão.

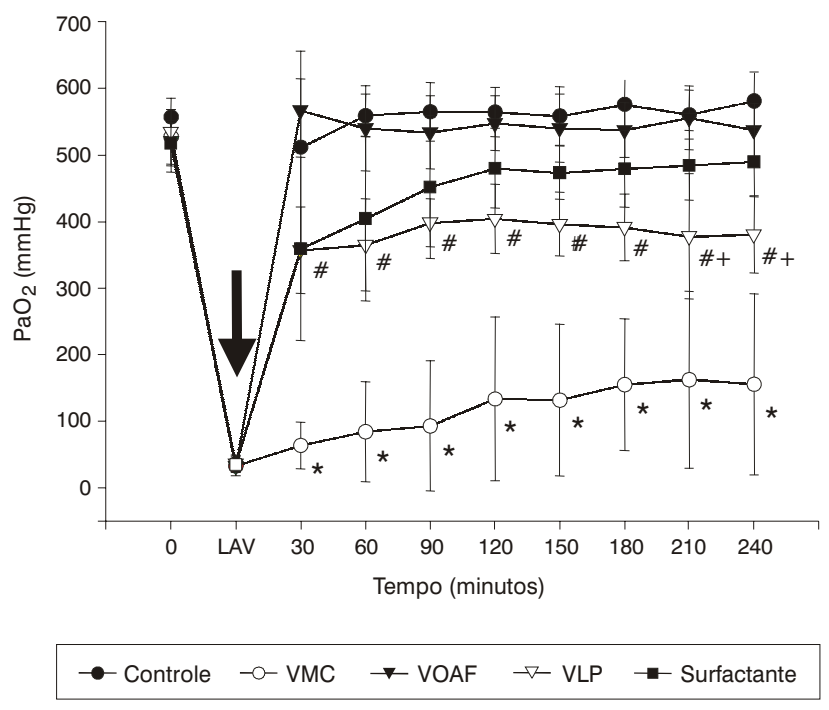

VMC: ventilação mecânica convencional; VOAF: ventilação oscilatória de alta freqüência; VLP: ventilação líquida parcial; LAV: lavagem traqueal (lesão). A seta indica uma redução significativa de $\mathrm{PaO}_{2}$ para todos os grupos em comparação com o estado basal $(p<0,05) \cdot{ }^{*} p<0,05$ em relação a todos os demais grupos. \#p $<0,05$ em relação aos grupos controle e VOAF. $+p<0,05$ em relação ao grupo surfactante.

Figura 2 - Medida de $\mathrm{PaO}_{2}$ para os diferentes grupos experimentais

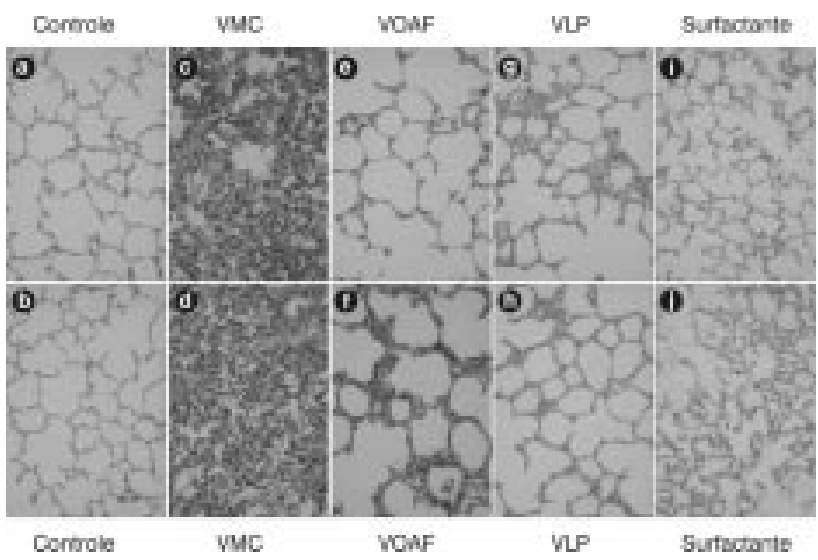

VMC: ventilação mecânica convencional; VOAF: ventilação oscilatória de alta freqüência; VLP: ventilação líquida parcial.

Figura 3 - Fotomicrografias digitais de microscopia óptica (200x, hematoxilina e eosina) representativas de amostras de pulmão de diferentes grupos experimentais, incluindo amostras das regiões não-dependentes (painel superior: a, c, e, g, i) e das regiões dependentes (painel inferior: $b, d, f, h, j$ )
Os resultados do escore objetivo de dano histopatológico global para os diferentes grupos experimentais são mostrados na Figura 4. Como esperado, amostras do grupo controle apresentaram mínima evidência de lesão. Em contraste, o grupo VMC demonstrou lesão significativamente maior do que os demais. Animais tratados com VOAF e VLP apresentaram baixos escores globais, ao contrário dos animais tratados com VMC-S.

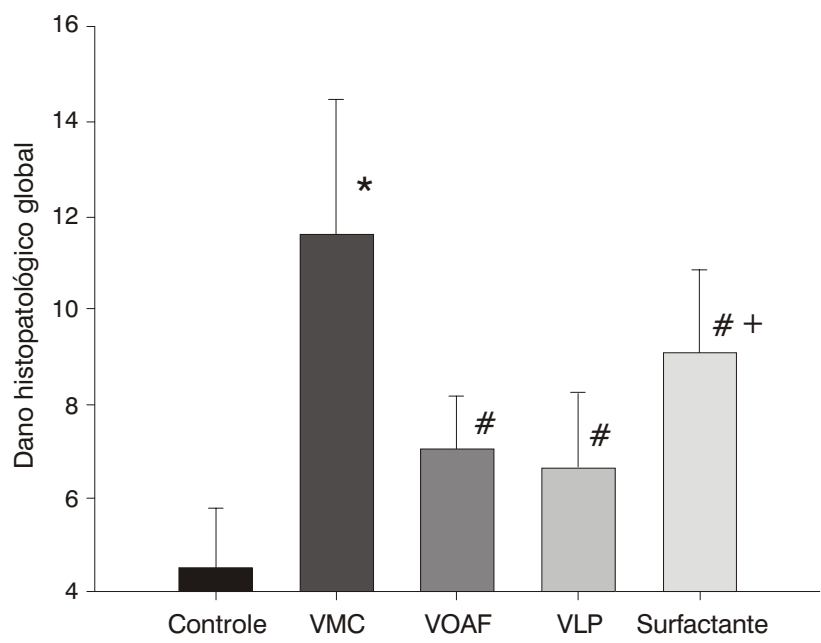

VMC: ventilação mecânica convencional; VOAF: ventilação oscilatória de alta freqüência; VLP: ventilação líquida parcial. * $p<0,05$ em comparação a todos os demais grupos. \#p <0,05 em relação ao grupo controle. $+p<0,05$ em relação aos grupos VOAF e VLP.

Figura 4 - Dano histopatológico global observado nos diferentes grupos experimentais

Os resultados do escore objetivo de dano histopatológico das regiões dependente e não-dependente para os diferentes grupos experimentais são mostrados na Figura 5 e confirmam a impressão subjetiva observada na Figura 3.

\section{Dano oxidativo}

As medidas dos níveis de 4-HNE, um marcador de dano oxidativo e indicador de peroxidação lipídica, são mostradas na Figura 6. O grupo tratado com VMC apresentou níveis significativamente mais elevados de 4-HNE em comparação com o grupo controle. Os níveis de 4-HNE nos grupos tratados com VLP e VOAF foram significativamente menores que no grupo VMC. O grupo VMC-S, apesar de apresentar níveis relativamente baixos de 4HNE, não demonstrou uma diferença estatisticamente significativa em relação ao grupo VMC.

\section{Discussão}

No presente estudo, foi utilizado um modelo experimental que envolve a combinação da infusão intravenosa de 

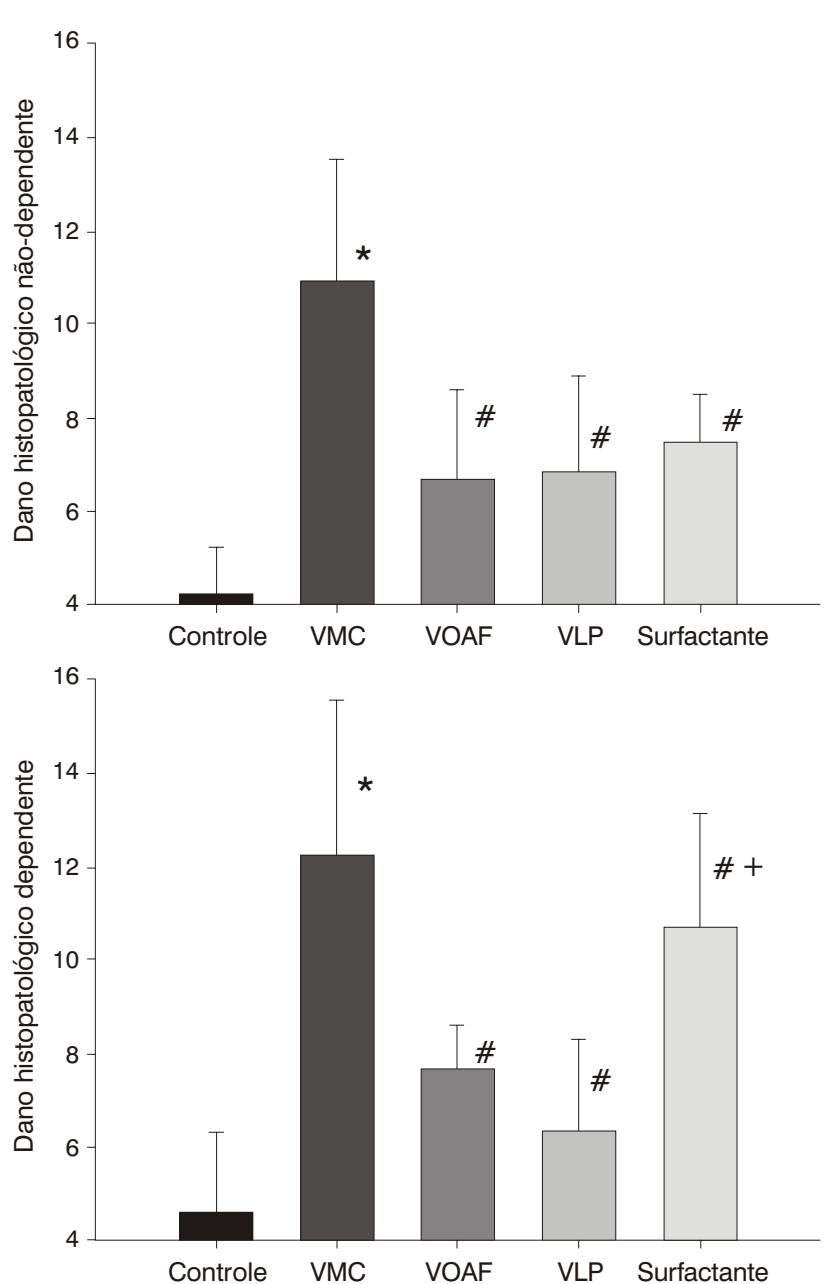

VMC: ventilação mecânica convencional; VOAF: ventilação oscilatória de alta freqüência; VLP: ventilação líquida parcial. * $p<0,05$ em comparação a todos os demais grupos. \#p $<0,05$ em relação ao grupo controle. $+p<0,05$ em relação aos grupos controle e VLP.

Figura 5 - Dano histopatológico dependente (painel inferior) e não-dependente (painel superior) observado nos diferentes grupos experimentais

endotoxina (lipopolissacarídeo) de E. coli e a lavagem pulmonar com solução salina para depleção de surfactante. O objetivo da metodologia em questão foi de proporcionar um modelo animal relevante ao estudo da SDRA em pacientes pediátricos, uma vez que cada um dos modelos isoladamente não proporciona ao investigador a possibilidade de estudar, em um curto espaço de tempo, as características inflamatórias e de alteração de complacência pulmonar inerentes à SDRA encontradas em ambiente clínico.

Isoladamente, o modelo de infusão intravenosa de endotoxina causa lesão pulmonar enquanto produz uma série de alterações análogas às observadas durante a septicemia avançada e o choque séptico em humanos ${ }^{21}$ causa mais freqüente de LPA e SDRA não-pulmonar em pacientes humanos ${ }^{22}$. Concomitantemente à característica de descompensação cardiovascular, a endotoxemia ativa uma série de sistemas em cascata, incluindo coagulação, fibrinólise e complemento ${ }^{23}$. Por sua vez, neutrófilos ativados pelo complemento são seqüestrados na microvasculatura pulmonar, onde a liberação de compostos reativos de oxigênio, proteases e citocinas inflamatórias são capazes de induzir e exacerbar a lesão do tecido pulmonar ${ }^{23,24}$.

A lavagem pulmonar com solução salina ${ }^{14}$ tem sido usada por uma série de investigadores por causar a depleção e disfunção do surfactante, bem como alterações de complacência pulmonar e hipoxemia análogas às que ocorrem na SDRA ${ }^{11,25}$. Uma crítica a esse modelo é que ele não envolve as alterações pró-inflamatórias sistêmicas encontradas na SDRA, uma condição experimental mitigada no presente estudo pela administração de endotoxina endovenosa para a ativação de neutrófilos circulantes antes da lavagem traqueal. O modelo de lavagem pulmonar causa uma previsível alteração de complacência no modelo de coelhos jovens. Em um estudo anterior, nosso laboratório demonstrou que três lavagens consecutivas com $30 \mathrm{ml} / \mathrm{kg}$ de solução salina são capazes de causar uma significativa e consistente alteração de complacência nesse modelo experimental, sendo que os animais desenvolviam um ponto de inflexão inferior na curva volume-pressão, em torno de 12 a $13 \mathrm{~cm}$ $\mathrm{H}_{2} \mathrm{O}^{11}$. Essa alteração era acompanhada de hipoxemia significativa, presumivelmente causada por colapso de unidades alveolares, uma vez que essa hipoxemia pode ser prontamente revertida com a aplicação de manobras de recrutamento pulmonar ${ }^{11}$.

No presente estudo, foram empregadas quatro diferentes estratégias de ventilação para suporte dos animais lesados: uma estratégia convencional não-protetora (VMC) e outras três conceptualmente protetoras (VMC-S, VLP e

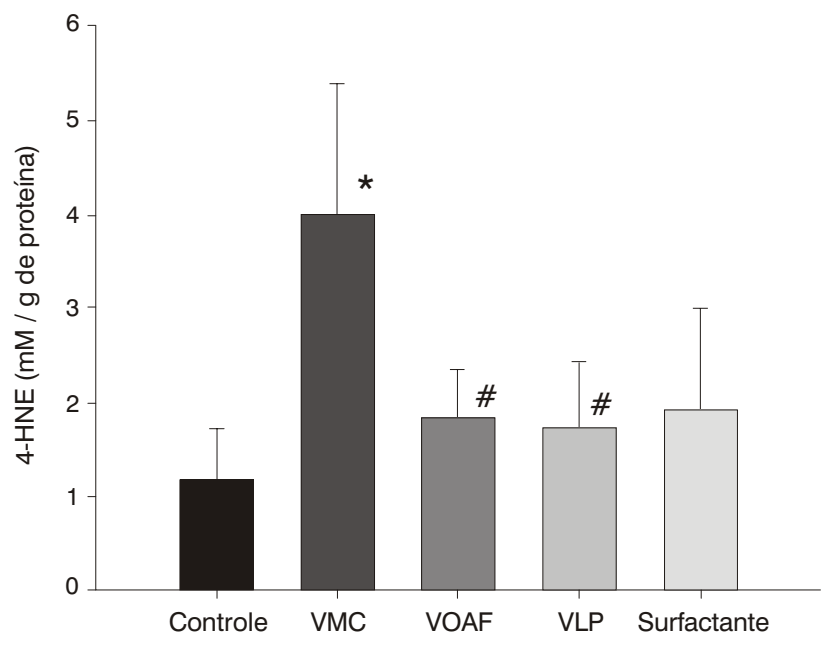

VMC: ventilação mecânica convencional; VOAF: ventilação oscilatória de alta freqüência; VLP: ventilação líquida parcial. * $\mathrm{p}<0,05$ em relação ao grupo controle. \#p < 0,05 em relação ao grupo VMC.

Figura 6 - Níveis de 4-HNE nos diversos grupos experimentais 
VOAF) por sua suposta capacidade de prevenir o atelectrauma e o agravamento da lesão pulmonar. O volume corrente de $10 \mathrm{ml} / \mathrm{kg}$ não resultou em evidência de hiperinsuflação alveolar (que poderia causar volutrauma), pois as pressões dinâmicas de pico inspiratório monitoradas pelo ventilador nunca excederam $25 \mathrm{~cm} \mathrm{H}_{2} \mathrm{O}$. O fato de que o mesmo volume corrente foi aplicado a todos os grupos, exceto ao de alta freqüência, propiciou uma comparação equilibrada do impacto das outras variáveis no resultado do experimento. Todos os animais incluídos no estudo desenvolveram hipoxemia de maneira previsível após a indução de lesão pulmonar. A forma aguda com que essas alterações ocorreram indica que elas são primariamente resultantes do processo de lavagem pulmonar ${ }^{11,14,25}$, e não da ativação de neutrófilos pela endotoxina, cujas alterações são menos abruptas $13,18,26$.

O decréscimo na $\mathrm{PaO}_{2}$ observado após a instituição da lesão pulmonar foi rapidamente revertido nos animais submetidos à VOAF. Entretanto, a mera iniciação de VOAF não garante a melhora da oxigenação, a não ser que uma pressão média de via aérea adequada seja empregada, como foi o caso no presente estudo. Por vezes, uma melhora significativa de oxigenação quando da iniciação da VOAF somente é observada após a aplicação de uma manobra de insuflação dinâmica sustentada, que tem o objetivo de propiciar recrutamento pulmonar rápido 11,25,27. No presente estudo, animais tratados com VLP apresentaram uma melhora rápida de oxigenação, mas $\mathrm{PaO}_{2}$ ficou limitada entre 350 e 400 torr durante o período experimental. Apesar de significativamente inferior à oxigenação apresentada pelo grupo tratado com VOAF, a $\mathrm{PaO}_{2}$ nos animais tratados com VLP mostrou-se compatível com aquela encontrada em outros modelos de lesão pulmonar aguda ${ }^{28}$. Cabe salientar que, mesmo quando utilizada em pulmões sadios, a VLP está associada a uma redução de $\mathrm{PaO}_{2}$, com níveis entre 350 e 450 torr, dependendo da espécie animal estudada 29,30 . Esse fenômeno é explicado por uma relativa barreira de difusão de oxigênio entre o perfluorocarbono e a superfície alveolar 29,30. Animais tratados com surfactante apresentaram melhora progressiva na oxigenação, embora com tendência a ser menor que a do grupo tratado com VOAF (sem significância estatística). O padrão de resposta da $\mathrm{PaO}_{2}$ nesse grupo sugere um recrutamento progressivo de unidades alveolares ao longo do experimento, apesar da probabilidade de que esses animais também sofreram colapso e reabertura cíclicos dos alvéolos mais dependentes, uma vez que a PEEP aplicada pareceu ser insuficiente para estabilizar os alvéolos durante o ciclo respiratório.

A importância da PEEP, mesmo nos animais tratados com surfactante, fica clara a partir do estudo de Hartog et al. ${ }^{31}$, onde animais lesados por lavagem pulmonar com solução salina foram ventilados com PEEP alta ou baixa antes do tratamento com surfactante exógeno ${ }^{31}$. Animais tratados com PEEP alta e surfactante apresentaram $\mathrm{PaO}_{2}$ significativamente maior do que animais tratados com PEEP baixa e surfactante. Considerando que, naquele estudo, animais com melhor oxigenação também apresentaram menores índices de proteína no lavado alveolar, é impossível discriminar o papel da atelectasia e da lesão pulmonar em comparação com a inativação de surfactante por proteínas plasmáticas na oxigenação de animais tratados com PEEP baixa 31,32 . Pode-se especular que, no presente estudo, a oxigenação do grupo tratado com surfactante poderia ter sido melhorada com o aumento da PEEP, buscando a manutenção do recrutamento pulmonar obtido e prevenindo atelectrauma. Entretanto, isso interferiria no objetivo de comparar o efeito de estratégias equivalentes com parâmetros ventilatórios semelhantes. No presente estudo, animais tratados com ventilação convencional mantiveram baixos níveis de oxigenação durante o período experimental. Esse achado não foi surpreendente, uma vez que normalmente se observa hipoxemia nesse modelo quando PEEPs inadequadamente baixas são empregadas ${ }^{11}$.

Como esperado, os animais sadios do grupo controle apresentaram baixos escores de lesão pulmonar. Os tratados com VMC demonstraram índices de dano histopatológico significativamente maiores que os demais grupos experimentais, de forma congruente com as medidas de oxigenação e ventilação. Esses animais apresentaram dano histológico envolvendo as regiões dependentes e nãodependentes do pulmão. De forma análoga ao que acontece na prática clínica, o escore de lesão da região dependente teve tendência a ser maior do que o observado na região não-dependente, apesar de esta observação não ter sido estatisticamente significativa. Animais tratados com VOAF e VLP apresentaram redução importante do escore de lesão histológica da região dependente em comparação com o grupo VMC e VMC-S. Esse achado confirma a impressão de que o grupo tratado com surfactante apresentou evidência de atelectrauma, presumivelmente pela aplicação de PEEP insuficiente. A tendência a um menor escore de lesão histológica na região dependente em animais tratados com VLP sugere que a alta concentração de perfluorocarbono nesse local possa estar associada a um maior efeito antiinflamatório local e proteção contra dano oxidativo tecidual ${ }^{28}$. O fato de os grupos surfactante, VLP e VOAF apresentarem escores de lesão histopatológica relativamente baixos na região não-dependente do pulmão não chega a surpreender, uma vez que essa região é a menos afetada, tanto na SDRA em humanos ${ }^{1,33}$ como em modelos experimentais ${ }^{11,18}$.

$A$ ativação e o seqüestro de neutrófilos no pulmão durante a fase inicial de lesão pulmonar aguda estão associados à produção de compostos reativos de oxigênio e ao conseqüente dano oxidativo ${ }^{34}$. O processo de peroxidação lipídica causa a destruição de lipídeos presentes na membrana celular, resultando na formação de peróxidos lipídicos e aldeídos, como o 4-HNE ${ }^{15,35}$. Em um estudo anterior, demonstramos uma elevação de 4-HNE em animais lesados com endotoxina intravenosa tratados com ventilação convencional, enquanto que animais tratados com VLP apresentaram níveis de 4-HNE atenuados ${ }^{16}$. No presente estudo, animais tratados com VMC apresentaram níveis elevados de 4-HNE, em contraste com o grupo controle. VLP e VOAF resultaram em níveis de 4-HNE significativamente mais baixos que os encontrados em animais tratados com VMC, demonstrando que aquelas estratégias, além de apresentarem menor lesão histopatológica, também estão associadas à redução do dano oxida- 
tivo pulmonar. Animais tratados com surfactante demonstraram níveis intermediários de 4-HNE, não sendo estatisticamente maiores do que os encontrados nos grupos VLP e VOAF, mas também não significativamente menores do que nos animas tratados com ventilação convencional.

Em conclusão, nesse modelo experimental pediátrico de SDRA, estratégias que evitam o atelectrauma, como VOAF e VLP, resultaram em oxigenação adequada, menor evidência de dano histológico e atenuação do dano oxidativo pulmonar em comparação com a VMC. A aplicação de surfactante exógeno proporciona uma melhor oxigenação em comparação com a VMC, mas resulta em lesão pulmonar aumentada, presumivelmente porque a PEEP inadequadamente baixa é insuficiente para estabilizar os alvéolos durante a expiração, causando atelectrauma.

\section{Referências}

1. Ware LB, Matthay MA. The acute respiratory distress syndrome. N Engl J Med. 2000;342:1334-49.

2. Delclaux C, L'Her E, Alberti C, Mancebo J, Abroug F, Conti G, et al. Treatment of acute hypoxemic nonhypercapnic respiratory insufficiency with continuous positive airway pressure delivered by a face mask: A randomized controlled trial. JAMA. 2000;284: 2352-60.

3. Suchyta MR, Clemmer TP, Orme JF Jr., Morris AH, Elliott CG. Increased survival of ARDS patients with severe hypoxemia (ECMO criteria). Chest. 1991;99:951-5.

4. Milberg JA, Davis DR, Steinberg KP, Hudson LD. Improved survival of patients with acute respiratory distress syndrome (ARDS): 1983-1993. JAMA. 1995;273:306-9.

5. Amato MB, Barbas CS, Medeiros DM, Magaldi RB, Schettino GP, Lorenzi-Filho $\mathrm{G}$, et al. Effect of a protective-ventilation strategy on mortality in the acute respiratory distress syndrome. $\mathrm{N}$ Engl J Med. 1998; 338:347-54.

6. ARDS-Network. Ventilation with lower tidal volumes as compared with traditional tidal volumes for acute lung injury and the acute respiratory distress syndrome. The Acute Respiratory Distress Syndrome Network. N Engl J Med. 2000;342:1301-8.

7. Hernandez LA, Peevy KJ, Moise AA, Parker JC. Chest wall restriction limits high airway pressure-induced lung injury in young rabbits. J Appl Physiol. 1989;66:2364-8.

8. Dreyfuss D, Soler P, Basset G, Saumon G. High inflation pressure pulmonary edema. Respective effects of high airway pressure, high tidal volume, and positive end-expiratory pressure. Am Rev Respir Dis. 1988;137:1159-64.

9. Slutsky AS. Lung injury caused by mechanical ventilation. Chest. 1999;116:S9-15.

10. Tremblay L, Valenza F, Ribeiro SP, Li J, Slutsky AS. Injurious ventilatory strategies increase cytokines and c-fos $m$-RNA expression in an isolated rat lung model. J Clin Invest. 1997; 99:944-52.

11. Rotta AT, Gunnarsson B, Fuhrman BP, Hernan LJ, Steinhorn DM. Comparison of lung protective ventilation strategies in a rabbit model of acute lung injury. Crit Care Med. 2001;29:2176-84.

12. ARDS-Network. Prospective, Randomized, Multi-Center Trial of Higher End-expiratory Lung Volume/Lower $\mathrm{FiO}_{2}$ versus Lower End-expiratory Lung Volume/Higher $\mathrm{FiO}_{2}$ Ventilation in Acute Lung Injury and Acute Respiratory Distress Syndrome [site na internet] Disponível: http://www.ardsnet.org/ards04.php. Acessado: 16 de Fevereiro de 2004.

13. Rotta AT, Steinhorn DM. Partial liquid ventilation reduces pulmonary neutrophil accumulation in an experimental model of systemic endotoxemia and acute lung injury. Crit Care Med. 1998;26:1707-15.

14. Lachmann B, Robertson B, Vogel J. In vivo lung lavage as an experimental model of the respiratory distress syndrome. Acta Anaesthesiol Scand. 1980;24:231-6.

15. Esterbauer $\mathrm{H}$, Cheeseman $\mathrm{KH}$. Determination of aldehydic lipid peroxidation products: malonaldehyde and 4-hydroxynonenal. Methods Enzymol. 1990;186:407-21.
16. Rotta AT, Gunnarsson B, Hernan LJ, Fuhrman BP, Steinhorn DM. Partial liquid ventilation with perflubron attenuates in vivo oxidative damage to proteins and lipids. Crit Care Med. $2000 ; 28: 202-8$

17. Mrozek JD, Smith KM, Bing DR, Meyers PA, Simonton SC, Connett $\mathrm{JE}$, et al. Exogenous surfactant and partial liquid ventilation: physiologic and pathologic effects. Am J Respir Crit Care Med. 1997; 156:1058-65.

18. Rotta AT, Gunnarsson B, Hernan LJ, Fuhrman BP, Steinhorn DM. Partial liquid ventilation influences pulmonary histopathology in an animal model of acute lung injury. J Crit Care. 1999;14:84-92.

19. Hernan LJ, Fuhrman BP, Papo MC, Leach CL, Thompson AE, Nesti FD, et al. Oxygenation during perfluorocarbon associated gas exchange in normal and abnormal lungs. Artif Cells Blood Substit Immobil Biotechnol. 1994;22:1377-80.

20. Hernan LJ, Fuhrman BP, Kaiser RE, Penfil S, Foley C, Papo MC, et al. Perfluorocarbon-associated gas exchange in normal and acid-injured large sheep. Crit Care Med. 1996;24:475-81.

21. Deitch EA. Animal models of sepsis and shock: a review and lessons learned. Shock. 1998;9:1-11.

22. Hudson LD, Milberg JA, Anardi D, Maunder RJ. Clinical risks for development of the acute respiratory distress syndrome. Am J Respir Crit Care Med. 1995;151:293-301.

23. Romero LA, Mariscalco MM, Fortenberry JD, Timmons OD. Effects of serum and endotoxin in experimental lung injury. J Pediatr Surg. 1990;25:846-9.

24. Worthen GS, Haslett C, Rees AJ, Gumbay RS, Henson JE, Henson PM. Neutrophil-mediated pulmonary vascular injury. Synergistic effect of trace amounts of lipopolysaccharide and neutrophil stimuli on vascular permeability and neutrophil sequestration in the lung. Am Rev Respir Dis. 1987; 136:19-28.

25. Sugiura M, McCulloch PR, Wren S, Dawson RH, Froese $A B$. Ventilator pattern influences neutrophil influx and activation in atelectasis-prone rabbit lung. J Appl Physiol. 1994;77:1355-65.

26. Fink MP, Heard SO. Laboratory models of sepsis and septic shock. J Surg Res. 1990;49:186-96.

27. Froese $A B$. High-frequency oscillatory ventilation for adult respiratory distress syndrome: let's get it right this time! Crit Care Med. 1997;25:906-8.

28. Fuhrman BP, Hernan LJ, Rotta A. Partial liquid ventilation: A multifaceted approach to acute respiratory distress syndrome. New Horiz. 1999; 7:433-9.

29. Fuhrman BP, Paczan PR, DeFrancisis M. Perfluorocarbonassociated gas exchange. Crit Care Med. 1991;19:712-22.

30. Hernan LJ, Penfil S, Fuhrman BP, Papo MC, Steinhorn DM, Leach $\mathrm{CL}$, et al. Functional $\mathrm{FiO}_{2}$ predicts alveolar $\mathrm{PO}_{2}$ during perfluorocarbon associated gas exchange. Crit Care Med. 1996;24:A149.

31. Hartog A, Gommers D, Haitsma JJ, Lachmann B. Improvement of lung mechanics by exogenous surfactant: effect of prior application of high positive end-expiratory pressure. $\mathrm{Br}$ J Anaesth. 2000;85:752-6.

32. Hartog A, Vazquez de Anda GF, Gommers D, Kaisers U, Lachmann B. At surfactant deficiency, application of "the open lung concept" prevents protein leakage and attenuates changes in lung mechanics. Crit Care Med. 2000;28:1450-4.

33. Gattinoni L, Pesenti A, Bombino M, Baglioni S, Rivolta M, Rossi $F$, et al. Relationships between lung computed tomographic density, gas exchange, and PEEP in acute respiratory failure. Anesthesiology. 1988;69:824-32.

34. Carey PD, Jenkins JK, Byrne K, Walsh CJ, Fowler AA, Sugerman $\mathrm{HJ}$. The neutrophil respiratory burst and tissue injury in septic acute lung injury: the effect of cyclooxygenase inhibition in swine. Surgery. 1992;112:45-55.

35. Demling $R$, LaLonde C. Relationship between lung injury and lung lipid peroxidation caused by recurrent endotoxemia. Am Rev Respir Dis. 1989;139:1118-24.

Correspondência:

Alexandre T. Rotta

Department of Anesthesiology

Driscoll Children's Hospital

3533 South Alameda Avenue

Corpus Christi, TX 78411, EUA

Tel.: +01 (361) 694.5445 - Fax: +01 (716) 8787101

E-mail: alexrotta@stx.rr.com 